\title{
A Riesz Representation Theorem for the Space of Henstock Integrable Vector-Valued Functions
}

\author{
Tomás Pérez Becerra $\mathbb{D}^{\mathrm{D}},{ }^{1}$ Juan Alberto Escamilla Reyna, ${ }^{1}$ Daniela Rodríguez Tzompantzi, ${ }^{1}$ \\ Jose Jacobo Oliveros Oliveros $\mathbb{D i D}^{1}{ }^{1}$ and Khaing Khaing Aye ${ }^{2}$ \\ ${ }^{1}$ Facultad de Ciencias Físico Matemáticas, Benemérita Universidad Autónoma de Puebla, Puebla, PUE, Mexico \\ ${ }^{2}$ Department of Engineering Mathematics, Yangon Technological University, Yangon, Myanmar \\ Correspondence should be addressed to Tomás Pérez Becerra; tompb55@hotmail.com
}

Received 15 January 2018; Revised 21 March 2018; Accepted 5 April 2018; Published 17 May 2018

Academic Editor: Adrian Petrusel

Copyright (C) 2018 Tomás Pérez Becerra et al. This is an open access article distributed under the Creative Commons Attribution License, which permits unrestricted use, distribution, and reproduction in any medium, provided the original work is properly cited.

\begin{abstract}
Using a bounded bilinear operator, we define the Henstock-Stieltjes integral for vector-valued functions; we prove some integration by parts theorems for Henstock integral and a Riesz-type theorem which provides an alternative proof of the representation theorem for real functions proved by Alexiewicz.
\end{abstract}

\section{Introduction}

Henstock in [1] defines a Riemann type integral which is equivalent to Denjoy integral and more general than the Lebesgue integral, called the Henstock integral. Cao in [2] extends the Henstock integral for vector-valued functions and provides some basic properties such as the SaksHenstock Lemma.

Schwabik in [3] considers a bilinear form, defines a Stieltjes type integral, and performs a study about it including [4]; following his ideas we give integration by parts theorem involving a bilinear operator and, through it, we prove a representation theorem for the space of Henstock vectorvalued functions.

This paper is divided into five sections; in a first step, in Section 2 we present some preliminaries and introduce the Henstock-Stieltjes integral via a bilinear bounded operator and the Bochner integral, together with some basic properties. In Section 3 we provide two useful kinds of integration by parts theorems, one of them in terms of the Bochner integral and the other using Henstock-Stieltjes integral; the representation theorem is proved in Section 4 which, if we consider real-valued functions, provides an alternative proof of the representation theorem proved by Alexiewicz (Theorem 1 in [5]).

\section{Preliminaries}

Throughout this paper $X, Y$, and $Z$ will denote three Banach spaces, $\|\cdot\|_{X},\|\cdot\|_{Y}$, and $\|\cdot\|_{Z}$, which will denote their respective norms, $X^{*}$ the dual of $X, B: X \times Y \rightarrow Z$ a bounded bilinear operator fixed, and $[a, b]$ a closed finite interval of the real line with the usual topology and the Lebesgue measure, which we denote by $\mu$. For a function $f:[a, b] \rightarrow \mathbb{R}$ we denote the Lebesgue integral of $f$ on a measurable $E \subset[a, b]$, when it exists, by $(L) \int_{E} f$.

Definition 1. $B: X \times Y \rightarrow Z$ is a bounded bilinear operator if $B$ is linear in each variable and there exists $M>0$ such that $\|B(x, y)\|_{Z} \leq M\|x\|_{X}\|y\|_{Y}$; in this case, the norm of the operator $B$ is $\|B\|=\inf \left\{M>0:\|B(x, y)\|_{Z} \leq M\|x\|_{X}\|y\|_{Y}\right\}$.

We say that $P=\left\{\left(\left[t_{i-1}, t_{i}\right], \xi_{i}\right): i=1, \ldots, n\right\}$ is a tagged partition of $[a, b]$ if $\left\{\left[t_{i-1}, t_{i}\right]: i=1, \ldots, n\right\}$ is a finite collection of nonoverlapping closed intervals whose union is $[a, b]$ such that $\xi_{i} \in\left[t_{i-1}, t_{i}\right]$ for every $i$. Given a function $\delta$ from $[a, b]$ to $(0, \infty)$, called gauge on $[a, b]$, we say that a tagged partition $\left\{\left(\left[t_{i-1}, t_{i}\right], \xi_{i}\right): i=1, \ldots, n\right\}$ is $\delta$-fine if

$$
\left[t_{i-1}, t_{i}\right] \subset\left(\xi_{i}-\delta\left(\xi_{i}\right), \xi_{i}+\delta\left(\xi_{i}\right)\right) \quad \text { for every } i
$$


Definition 2. A function $f:[a, b] \rightarrow X$ is Kurzweil integrable in $[a, b]$ if there exists $w \in X$ such that for every $\epsilon>0$ there exists a gauge $\delta$ on $[a, b]$ such that if $\left\{\left(\left[t_{i-1}, t_{i}\right], \xi_{i}\right): i=\right.$ $1, \ldots, n\}$ is a $\delta$-fine tagged partition of $[a, b]$, then

$$
\left\|\sum_{i=1}^{n} f\left(\xi_{i}\right)\left[t_{i}-t_{i-1}\right]-w\right\|_{X}<\epsilon .
$$

We write $w=(K) \int_{a}^{b} f$.

Definition 3. A function $f:[a, b] \rightarrow X$ is Henstock integrable in $[a, b]$ if there exists $F:[a, b] \rightarrow X$ such that for every $\epsilon>0$ there exists a gauge $\delta$ on $[a, b]$ such that if $\left\{\left(\left[t_{i-1}, t_{i}\right], \xi_{i}\right)\right.$ : $i=1, \ldots, n\}$ is a $\delta$-fine tagged partition of $[a, b]$, then

$$
\sum_{i=1}^{n}\left\|f\left(\xi_{i}\right)\left[t_{i}-t_{i-1}\right]-\left[F\left(t_{i}\right)-F\left(t_{i-1}\right)\right]\right\|_{X}<\epsilon .
$$

We write $F(b)-F(a)=(H) \int_{a}^{b} f$.

The Henstock integral is also known as HenstockLebesgue integral, briefly HL integral ([6]), or variational Henstock integral ([7]).

In [8] we can find some properties of both integrals such as the linearity, integrability over subintervals, and the continuity of the function $F:[a, b] \rightarrow X$, called primitive, given by $F(t)=(H) \int_{a}^{t} f$ or $F(t)=(K) \int_{a}^{t} f, t \in[a, b]$.

Definition 4. Let $F:[a, b] \rightarrow X$ and let $E$ be a subset of $[a, b]$.

(1) $F$ is said to be of strongly bounded variation (BV) on $E$ if the number $V(F, E):=\sup \left\{\sum_{i}\left\|F\left(d_{i}\right)-F\left(c_{i}\right)\right\|_{X}\right\}$ is finite, where the supremum is taken over all finite sequences $\left\{\left[c_{i}, d_{i}\right]\right\}$ of nonoverlapping intervals that have endpoints in $E$.

(2) $F$ is $\mathrm{BV}^{*}$ on $E$ if $\sup \left\{\sum_{i} \omega\left(F,\left[c_{i}, d_{i}\right]\right)\right\}$ is finite, where the supremum is taken over all finite sequences $\left\{\left[c_{i}, d_{i}\right]\right\}$ of nonoverlapping intervals that have endpoints in $E$, and $\omega\left(F ;\left[c_{i}, d_{i}\right]\right)=\sup \{\|F(y)-F(x)\|$ : $\left.x, y \in\left[c_{i}, d_{i}\right]\right\}$ is the oscillation of $F$ on $\left[c_{i}, d_{i}\right]$.

(3) $F$ is said to be strongly absolutely continuous on $E$ or $\mathrm{AC}(E)$ if for every $\epsilon>0$ there exists $\eta>0$ such that, for every finite or infinite sequence of nonoverlapping intervals $\left\{\left[c_{i}, d_{i}\right]\right\}$, with $\sum_{i}\left(d_{i}-c_{i}\right)<\eta$, we have $\sum_{i}\left\|F\left(d_{i}\right)-F\left(c_{i}\right)\right\| \leq \epsilon$ where $c_{i}, d_{i} \in E$ for all $i$.

(4) $F$ is $\operatorname{AC}^{*}(E)$ if for every $\epsilon>0$ there exists $\eta>$ 0 such that, for every finite or infinite sequence or nonoverlapping intervals $\left\{\left[c_{i}, d_{i}\right]\right\}$ satisfying $\sum_{i}\left(d_{i}-\right.$ $\left.a_{i}\right)<\eta$, where $c_{i}, d_{i} \in E$ for all $i$, we have $\sum_{i} \omega\left(F ;\left[c_{i}, d_{i}\right]\right)<\epsilon$.

(5) $F$ is $\mathrm{ACG}^{*}$ on $E$ if $E$ is the union of a sequence of closed sets $\left\{E_{i}\right\}$ such that, on each $E_{i}, F$ is $\operatorname{AC}^{*}\left(E_{i}\right)$.

The next result gives us a characterization of Henstock integrability.
Theorem 5 (see [8, Thm. 7.4.5, pp. 217]). The function $f$ : $[a, b] \rightarrow X$ is Henstock integrable on $[a, b]$ with the primitive $F$ if and only if $F:[a, b] \rightarrow X$ is continuous and $A C G^{*}$ on $[a, b]$ such that $F^{\prime}(t)=f(t)$ almost everywhere (a.e.) in $[a, b]$, where the derivative is in the sense of Frechet.

If a function $F:[a, b] \rightarrow X$ is $\mathrm{BV}$ or $\mathrm{BV}^{*}$ on $E$, then it is bounded on $E$; that is, $K>0$ exists such that $\|F(t)\|_{X} \leq K$, for every $t \in E$. As an $\mathrm{AC}(E)$ function is $\mathrm{BV}$ on $E$ and an $\mathrm{AC}^{*}(E)$ function is $\mathrm{BV}^{*}$ on $E$ (immediately from the definitions), then every $\mathrm{AC}(E)$ or $\mathrm{AC}^{*}(E)$ function is also bounded in $E$. It is easy to see that if $F$ is $\mathrm{AC}(E)$ and $E_{0} \subset E$, then $F$ is $\operatorname{AC}\left(E_{0}\right)$, similarly if $F$ is $\mathrm{AC}^{*}(E)$.

The definition of a function of strongly bounded variation can be extended considering the bilinear operator $B: X \times$ $Y \rightarrow Z$.

Definition 6. Let $G:[a, b] \rightarrow Y$ be a function and $D=$ $\left\{t_{0}, t_{1}, \ldots, t_{n}\right\}$ a partition of $[a, b]$; we define

$$
V_{a}^{b}(G, D)=\sup \left\{\sum_{i=1}^{n}\left\|B\left(x_{i}, G\left(t_{i}\right)-G\left(t_{i-1}\right)\right)\right\|_{Z}\right\},
$$

where the supremum is taken over all possible elections of $x_{i} \in X, i=1,2, \ldots, n$, with $\left\|x_{i}\right\|_{X} \leq 1$.

$$
(\mathfrak{B}) \operatorname{var}_{a}^{b}(G)=\sup \left\{V_{a}^{b}(G, D)\right\},
$$

where the supremum is taken over all partitions of the interval $[a, b]$ and $(\mathfrak{B}) \operatorname{var}_{a}^{b}(G)$ is the strong $\mathfrak{B}$-variation of $G$ on $[a, b]$. If we consider

$$
\widehat{V}_{a}^{b}(G, D)=\sup \left\{\left\|\sum_{i=1}^{m} B\left(x_{i}, G\left(t_{j}\right)-G\left(t_{j-1}\right)\right)\right\|_{Z}\right\}
$$

in the equality (4), then we define $(\mathfrak{B}) \widehat{\operatorname{var}}_{a}^{b}(G)=\sup \left\{\widehat{V}_{a}^{b}(G\right.$, $D)\}$ as the $\mathfrak{B}$-variation of $G$ on $[a, b]$.

If $(\mathfrak{B}) \operatorname{var}_{a}^{b}(G)<\infty$ or $(\mathfrak{B}) \widehat{\operatorname{var}}_{a}^{b}(G)<\infty$ we say that $G$ is of strongly bounded $\mathfrak{B}$-variation or $G$ is of bounded $\mathfrak{B}$-variation, respectively.

It is straightforward that each function of strongly bounded variation is of strongly bounded $\mathfrak{B}$-variation. We recommend the reader interested in this topic to consult the study exposed in [9].

2.1. Stieltjes-Type Integrals. As we mentioned in the introduction, Schwabik in [3] gives the next definition and proves some basic properties such as the Uniform Convergence Theorem.

Definition 7. $I \in Z$ is the Kurzweil-Stieltjes integral of $f$ : $[a, b] \rightarrow X$ with respect to $g:[a, b] \rightarrow Y$ if for every $\epsilon>0$ there exists a gauge $\delta$ on $[a, b]$ such that

$$
\left\|\sum_{i=1}^{n} B\left(f\left(\xi_{i}\right), g\left(t_{i}\right)-g\left(t_{i-1}\right)\right)-I\right\|_{Z}<\epsilon,
$$

for every $\delta$-fine tagged partition $\left\{\left(\left[t_{i-1}, t_{1}\right], \xi_{i}\right): i=1, \ldots, n\right\}$ of $[a, b]$.

In this case we write $I=(K S) \int_{a}^{b} B(f, d g)$. 
Now, we introduce the following integral.

Definition 8. A function $f:[a, b] \rightarrow X$ is Henstock-Stieltjes integrable in $[a, b]$ with respect to $g:[a, b] \rightarrow Y$ if there exists $H:[a, b] \rightarrow Z$ such that for every $\epsilon>0$ there exists a gauge $\delta$ of $[a, b]$ such that if $\left\{\left(\left[t_{i-1}, t_{i}\right], \xi_{i}\right): i=1, \ldots, n\right\}$ is a $\delta$-fine tagged partition of $[a, b]$, then

$$
\begin{aligned}
& \sum_{i=1}^{n}\left\|B\left(f\left(\xi_{i}\right), g\left(t_{i}\right)-g\left(t_{i-1}\right)\right)-\left[H\left(t_{i}\right)-H\left(t_{i-1}\right)\right]\right\|_{Z} \\
& \quad<\epsilon .
\end{aligned}
$$

We write $H(b)-H(a)=(H S) \int_{a}^{b} B(f, d g)$.

It is immediate that every Henstock integrable function is Kurzweil integrable and its integrals are the same; we can repeat the proof of this fact for the previous Stieltjes integrals. Similarly, we can prove the properties of linearity and integrability over subintervals for the Henstock-Stieltjes integral directly of the proofs in [8] with slight changes. We omit the formulations and the proofs of such results.

Theorem 9 (see [3, Thm. 11]). Assume that the functions $f$, $f_{n}:[a, b] \rightarrow X$, and $g:[a, b] \rightarrow Y$ are given. If $(\mathfrak{B}) \widehat{\operatorname{var}}_{a}^{b}(g)<$ $\infty$, the Kurzweil-Stieltjes integrals $(K S) \int_{a}^{b} B\left(f_{n}, d g\right)$ exist and the sequence $\left\{f_{n}\right\}$ converges on $[a, b]$ uniformly to $f$, then the integral $(K S) \int_{a}^{b} B(f, d g)$ exists and

$$
(K S) \int_{a}^{b} B\left(f_{n}, d g\right)=\lim _{n \rightarrow \infty}(K S) \int_{a}^{b} B(f, d g) .
$$

2.2. Bochner Integral. Let us recall that a function $s:[a, b] \rightarrow$ $X$ is called simple if there is a finite sequence $\left\{E_{m}\right\}_{m=1}^{p} \subset[a, b]$ of Lebesgue measurable sets such that $E_{m} \cap E_{l}=\emptyset$ for $m \neq l$ and $[a, b]=\bigcup_{m=1}^{p} E_{m}$, where $s(t)=x_{m} \in X$ for $t \in E_{m}$, $m=1, \ldots, p$, and in this case the Bochner integral of $s$ is $(\mathfrak{B}) \int_{a}^{b} s=\sum_{m} x_{m} \mu\left(E_{m}\right)$.

A function $f:[a, b] \rightarrow X$ is strongly measurable if there exists a sequence of simple functions that converges pointwise to $f$ a.e. on $[a, b]$.

A function $f:[a, b] \rightarrow X$ is Bochner integrable if there is a sequence of simple functions $f_{n}:[a, b] \rightarrow X, n \in \mathbb{N}$, such that $\lim _{n \rightarrow \infty} f_{n}(t)=f(t)$ a.e. in $[a, b]$ and

$$
\lim _{n}(L) \int_{a}^{b}\left\|f_{n}-f\right\|=0 ;
$$

the Bochner integral of $f:[a, b] \rightarrow X$ is denoted by $(\mathscr{B}) \int_{a}^{b} f$ and is defined by

$$
(\mathscr{B}) \int_{a}^{b} f=\lim _{n}(\mathscr{B}) \int_{a}^{b} f_{n} .
$$

We will use the following well-known results of the Bochner integral.
Theorem 10 (see [8, Cor. 1.4.4, pp. 26]). A strongly measurable function $f:[a, b] \rightarrow X$ is Bochner integrable on $[a, b]$ if there exists a function $g:[a, b] \rightarrow \mathbb{R}$, which is Lebesgue integrable such that $\|f(t)\|_{X} \leq g(t), t \in[a, b]$.

Theorem 11 (see [8, Thm. 7.4.5, pp. 222]). A function $f$ : $[a, b] \rightarrow X$ is Bochner integrable on $[a, b]$ if and only if there exists a function $F:[a, b] \rightarrow X$, which is $A C$ on $[a, b]$ such that $F^{\prime}(t)=f(t)$ a.e. on $[a, b]$.

Given $F:[a, b] \rightarrow X$ and $g:[a, b] \rightarrow Y$ we define the function $B(F, g):[a, b] \rightarrow Z$, given by $B(F, g)(t)=$ $B(F(t), g(t))$; we will use this function from now on.

Lemma 12. If $F:[a, b] \rightarrow X$ is a continuous function and $g:[a, b] \rightarrow Y$ is Bochner integrable, then the function $B(F, g)$ is Bochner integrable.

Proof. Since $F$ is continuous, then it is strongly measurable; moreover there exists $K>0$ such that $\|F(t)\|_{X} \leq K$ for all $t \epsilon$ $[a, b]$. Because $g$ is strongly measurable and $B$ is continuous, $B(F, g)$ is strongly measurable.

By Theorem 10 the real function $\|g(t)\|_{Y}$ is Lebesgue integrable.

Now

$$
\begin{aligned}
\|B(F, g)(t)\|_{Z} & \leq\|B\|\|F(t)\|_{X}\|g(t)\|_{Y} \\
& \leq K\|B\|\|g(t)\|_{Y} ;
\end{aligned}
$$

hence $B(F, g)$ is Bochner integrable as a consequence of Theorem 10 .

It is known that every vector-valued function which is strongly measurable is weakly measurable; that is, $x^{*} F$ : $[a, b] \rightarrow \mathbb{R}$ is measurable for each $x^{*} \in X^{*}$; the inverse, in general, is not true (see [10, Example 5, Chapter II, \$1, and pp. 43]) however under certain conditions is equivalent.

Theorem 13 (see [10, Thm. 2, Chapter II, \$1, pp. 42] (Pettis)). Let $F:[a, b] \rightarrow X$ be a function. The following conditions are equivalent:

(i) F is strongly measurable.

(ii) $F$ is weakly measurable and there exists a measurable set $E \subset[a, b]$ with $\mu([a, b]-E)=0$ such that $F(E)$ is separable.

Theorem 14. If $F:[a, b] \rightarrow X$ is continuous a.e., then $F$ is strongly measurable.

Proof. As $F$ is continuous a.e., then $F$ is weakly continuous; that is, $x^{*} F:[a, b] \rightarrow \mathbb{R}$ is continuous a.e. and, hence, measurable.

We define $E=\{t \in[a, b]: F(t)$ is continuous $\}$. $E$ is Lebesgue measurable with $\mu([a, b]-E)=0$, as $[a, b]$ is separable and then $E$ is separable and, furthermore, $F$ is continuous, and then $F(E)$ is separable; hence $F$ is strongly measurable by the Pettis Theorem. 
Lemma 15 (see [11, Lemma 6]). If $g:[a, b] \rightarrow X$ is of strongly bounded variation on $[a, b]$, then $g$ is Bochner integrable on $[a, b]$.

As a consequence of Lemmas 12 and 15, we have the following result.

Corollary 16. Let $f:[a, b] \rightarrow X$ be Henstock integrable on $[a, b]$ and $F$ its primitive, $g:[a, b] \rightarrow Y$ of strongly bounded variation, then $B(F, g)$ is Bochner integrable on $[a, b]$.

It is easy to prove that the set of functions $\mathrm{AC}, \mathrm{AC}^{*}, \mathrm{ACG}$, and $\mathrm{ACG}^{*}$ on $E \subset[a, b]$ form vector spaces with the sum and product by scalars; moreover, these spaces of functions are algebras under the bilinear operator $B$.

Lemma 17. Let $F:[a, b] \rightarrow X$ and $G:[a, b] \rightarrow X$ be functions and $E$ a subset of $[a, b]$. If $F$ is $A C G^{*}(E)$ and $G$ is $A C(E)$, then $F+G$ is $A C G^{*}(E)$.

Proof. Since $F$ is $\operatorname{ACG}^{*}(E)$ then $E=\bigcup_{n} E_{n}$, where $F$ is $\operatorname{AC}^{*}\left(E_{n}\right)$.

For every $\epsilon>0$ there exists $\eta>0$ such that, for every $\left\{\left[c_{i}, d_{i}\right]: c_{i}, d_{i} \in E_{n}\right\}$, with $\sum_{i}\left(d_{i}-c_{i}\right)<\eta$, we have $\sum_{i} \omega(F$; $\left.\left[c_{i}, d_{i}\right]\right)<\epsilon$ and $\sum_{i}\left\|G\left(d_{i}\right)-G\left(c_{i}\right)\right\|_{X}<\epsilon$, and

$$
\begin{aligned}
& \left\|F\left(d_{i}\right)+G\left(d_{i}\right)-F\left(c_{i}\right)-G\left(c_{i}\right)\right\|_{X} \\
& \quad \leq\left\|F\left(d_{i}\right)-F\left(c_{i}\right)\right\|_{X}+\left\|G\left(d_{i}\right)-G\left(c_{i}\right)\right\|_{X},
\end{aligned}
$$

and then $\sum_{i} \omega\left(F+G ;\left[c_{i}, d_{i}\right]\right)<2 \epsilon$.

Lemma 18. Let $F:[a, b] \rightarrow X$ and $g:[a, b] \rightarrow Y$ be functions and $E$ a subset of $[a, b]$. If $F$ is $A C G^{*}(E)$ and $g$ is $A C(E)$, then $B(F, g)$ is $A C G^{*}(E)$.

Proof. The proof is analogous to Lemma 17 changing the inequality 2 by

$$
\begin{gathered}
\left\|B\left(F\left(d_{i}\right), g\left(d_{i}\right)\right)-B\left(F\left(c_{i}\right), g\left(c_{i}\right)\right)\right\|_{Z} \\
\leq\|B\|\left\|F\left(d_{i}\right)-F\left(c_{i}\right)\right\|_{X}\left\|g\left(d_{i}\right)\right\|_{Y} \\
+\|B\|\left\|F\left(c_{i}\right)\right\|_{X}\left\|g\left(d_{i}\right)-g\left(c_{i}\right)\right\|_{Y} .
\end{gathered}
$$

\section{Integration by Parts Theorem}

\subsection{Involving Bochner Integral}

Theorem 19. Let $f:[a, b] \rightarrow X$ be a Henstock integrable function with primitive $F, g:[a, b] \rightarrow Y$ of strongly bounded variation, and $G$ the Bochner primitive of $g$. Then $(H) \int_{a}^{b} B(f$, $G)$ exists and

$$
(H) \int_{a}^{b} B(f, G)=B(F(b), G(b))-(\mathscr{B}) \int_{a}^{b} B(F, g) \text {. }
$$

Proof. By Corollary $16, B(F, g)$ is Bochner integrable. Let $\phi$ : $[a, b] \rightarrow Z$ be a function given by

$$
\phi(t)=B(F(t), G(t))-(\mathscr{B}) \int_{a}^{t} B(F, g) .
$$

$\phi$ is continuous due to the continuity on $[a, b]$ of $F, G$, and ( B) $\int_{a}^{t} B(F, g)$.

Theorem 5 implies that $F$ is $\mathrm{ACG}^{*}$ on $[a, b]$ and Theorem 11 implies that $G$ is $\mathrm{AC}$ on $[a, b]$; hence the function $t \rightarrow B(F(t), G(t))$ is $\mathrm{ACG}^{*}$ on $[a, b]$ by Lemma 18 . Finally, Lemma 17 implies that $\phi$ is $\mathrm{ACG}^{*}$ on $[a, b]$.

In order to prove that $\phi$ is differentiable on $[a, b]$, using the fact that $B(F(t) /(s-t), G(s))=B(F(t), G(s) /(s-t))$ for every $s, t \in[a, b]$, we calculate

$$
\begin{aligned}
& \| \frac{B(F(s), G(s))-B(F(t), G(t))}{s-t}-B(F(t), g(t)) \\
& -B(f(t), G(t))\left\|_{Z} \leq\right\| B\|\| \frac{F(s)-F(t)}{s-t}-f(t) \|_{X} \\
& \cdot\|G(s)\|_{Y}+\|B\|\|F(t)\|_{X}\left\|\frac{G(t)-G(s)}{s-t}-g(t)\right\|_{Y} \\
& +\|B\|\|f(t)\|_{X}\|G(s)-G(t)\|_{Y},
\end{aligned}
$$

which tends to 0 when $s \rightarrow t$; hence $B(F, G)$ is differentiable on $[a, b]$ and $(B(F, G))^{\prime}=B(F, g)+B(f, G)$. Then $\phi^{\prime}(t)=$ $(B(F, G))^{\prime}-B(F, g)=B(F, g)+B(f, G)-B(F, g)=B(f, G)$. By Theorem $5,(H) \int_{a}^{b} B(f, G)$ exists and (15) is fulfilled.

\subsection{Involving Henstock-Stieltjes Integral}

Theorem 20. Let $f:[a, b] \rightarrow X$ and $g:[a, b] \rightarrow Y$ be functions. If the integral (HS) $\int_{a}^{b} B(f, d g)$ exists and $(\mathfrak{B}) \operatorname{var}_{a}^{b}(g)<$ $\infty$, then

$$
\left\|(H S) \int_{a}^{b} B(f, d g)\right\|_{Z} \leq \sup _{t \in[a, b]}\|f(t)\|_{X}(\mathfrak{B}) \operatorname{var}_{a}^{b}(g) .
$$

Proof. Let $\epsilon>0$. There exists a gauge $\delta$ on $[a, b]$ such that

$$
\begin{aligned}
\sum_{i=1}^{n} \| & B\left(f\left(\xi_{i}\right), g\left(t_{i}\right)-g\left(t_{i-1}\right)\right)-(H S) \int_{t_{i-1}}^{t_{i}} B(f, d g) \|_{Z} \\
& <\epsilon,
\end{aligned}
$$

for every $P=\left\{\left(\left[t_{i-1}, t_{i}\right], \xi_{i}\right): i=1, \ldots, n\right\}$, which is $\delta$-fine. Then,

$$
\begin{gathered}
\left\|(H S) \int_{a}^{b} B(f, d g)\right\|_{Z} \leq \|(H S) \int_{a}^{b} B(f, d g) \\
-\sum_{i=1}^{n} B\left(f\left(\xi_{i}\right), g\left(t_{i}\right)-g\left(t_{i-1}\right)\right) \\
+\sum_{i=1}^{n} B\left(f\left(\xi_{i}\right), g\left(t_{i}\right)-g\left(t_{i-1}\right)\right) \|_{Z} \leq \epsilon
\end{gathered}
$$




$$
\begin{aligned}
& +\sum_{i=1,\left\|f\left(\xi_{i}\right)\right\| \neq 0}^{n} \| B\left(\frac{f\left(\xi_{i}\right)\left\|f\left(\xi_{i}\right)\right\|_{X}}{\left\|f\left(\xi_{i}\right)\right\|_{X}}, g\left(t_{i}\right)\right. \\
& \left.-g\left(t_{i-1}\right)\right)\left\|_{Z} \leq \epsilon+\sup _{t \in[a, b]}\right\| f(t) \|_{X}(\mathfrak{B}) \operatorname{var}_{a}^{b}(g) .
\end{aligned}
$$

Now, we shall prove the following Theorem, which is a consequence of Theorem 9.

Theorem 21 (uniform convergence theorem). Let $f, f_{n}$ : $[a, b] \rightarrow X, n=1,2, \ldots$, and $g:[a, b] \rightarrow Y$. If $(\mathfrak{B}) \operatorname{var}_{a}^{b}(g)<$ $\infty$, the integrals (HS) $\int_{a}^{b} B\left(f_{n}, d g\right)$ exist for each $n$, and the sequence $f_{n}$ converges uniformly to $f$ in $[a, b]$, then the integral (HS) $\int_{a}^{b} B(f, d g)$ exists and

$$
(H S) \int_{a}^{b} B(f, d g)=\lim _{n \rightarrow \infty}(H S) \int_{a}^{b} B\left(f_{n}, d g\right) .
$$

Proof. Let $\epsilon>0$; because $f_{n}$ converges uniformly to $f$, there exists $n_{0}>0$ such that for every $n>n_{0}$ and $t \in[a, b]$

$$
\left\|f_{n}(t)-f(t)\right\|_{X}<\epsilon .
$$

Hence, for every $m, n>n_{0},\left\|f_{n}(t)-f_{m}(t)\right\|_{X} \leq 2 \epsilon$.

Theorem 9 implies the existence of the integral $H(J)=$ (HS) $\int_{J} B(f, d g)$ for every $J \subset[a, b]$ and $\lim _{n \rightarrow \infty} H_{n}(J)=$ $H(J)$. Hence, there exists $n_{1} \in \mathbb{N}$ such that $\left\|H_{n}(J)-H(J)\right\|_{Z}<$ $\epsilon$, for every $n>n_{1}$.

Let $m>\max \left\{n_{0}, n_{1}\right\}$ be fixed; as the integral

$$
(H S) \int_{a}^{b} B\left(f_{m}, d g\right):=H_{m}(b)-H_{m}(a)
$$

exists, there exists a gauge $\delta$ on $[a, b]$ such that, for every $\delta$ fine tagged partition $\left\{\left(\left[t_{i-1}, t_{i}\right], \xi_{i}\right): i=1, \ldots, n\right\}$ of $[a, b]$,

$$
\begin{gathered}
\sum_{i=1}^{n} \| B\left(f_{m}\left(\xi_{i}\right), g\left(t_{i}\right)-g\left(t_{i-1}\right)\right) \\
-(H S) \int_{t_{i-1}}^{t_{1}} B\left(f_{m}, d g\right) \|_{Z}<\epsilon .
\end{gathered}
$$

We have

$$
\begin{aligned}
& \sum_{i=1}^{n}\left\|B\left(f\left(\xi_{i}\right), g\left(t_{i}\right)-g\left(t_{i-1}\right)\right)-\left[H\left(t_{i}\right)-H\left(t_{i-1}\right)\right]\right\|_{Z} \\
& \quad \leq \sum_{i=1}^{n} \| B\left(f\left(\xi_{i}\right), g\left(t_{i}\right)-g\left(t_{i-1}\right)\right)-B\left(f_{m}\left(\xi_{i}\right), g\left(t_{i}\right)\right. \\
& \left.\quad-g\left(t_{i-1}\right)\right)\left\|_{Z}+\sum_{i=1}^{n}\right\| B\left(f_{m}\left(\xi_{i}\right), g\left(t_{i}\right)-g\left(t_{i-1}\right)\right)-(H S) \\
& \quad \cdot \int_{t_{i-1}}^{t_{1}} B\left(f_{m}, d g\right)\left\|_{Z}+\sum_{i=1}^{n}\right\|(H S) \int_{t_{i-1}}^{t_{1}} B\left(f_{m}, d g\right)-\left[H\left(t_{i}\right)\right. \\
& \left.\quad-H\left(t_{i-1}\right)\right] \|_{Z}
\end{aligned}
$$

$$
\begin{aligned}
& \leq \sum_{i, f\left(\xi_{i}\right) \neq f_{m}\left(\xi_{i}\right)} \| B\left(\frac{\left[f\left(\xi_{i}\right)-f_{m}\left(\xi_{i}\right)\right]\left\|f\left(\xi_{i}\right)-f_{m}\left(\xi_{i}\right)\right\|_{X}}{\left\|f\left(\xi_{i}\right)-f_{m}\left(\xi_{i}\right)\right\|_{X}},\right. \\
& \left.g\left(t_{i}\right)-g\left(t_{i-1}\right)\right)\left\|_{Z}+2 \epsilon \leq \sup _{t \in[a, b]}\right\| f(t)-f_{m}(t) \|_{X}(\mathfrak{B}) \\
& \cdot \operatorname{var}_{a}^{b}(g)+2 \epsilon \leq 3 \epsilon .
\end{aligned}
$$

Hence, $(H S) \int_{a}^{b} B(f, d g)$ exists and

$$
(H S) \int_{a}^{b} B(f, d g)=\lim _{n \rightarrow \infty}(H S) \int_{a}^{b} B\left(f_{n}, d g\right) .
$$

Theorem 22. If $f:[a, b] \rightarrow X$ is a step function, then for every $g:[a, b] \rightarrow Y$, the integral $(H S) \int_{a}^{b} B(f, d g)$ exists.

Proof. Analogous to the proof of [12, Lemma 3.2], is enough to prove for functions of the forms $\chi_{[a, \tau]} x, \chi_{[\tau, b]} x, \chi_{[a]} x$, and $\chi_{[b]} x$, where $\tau \in(a, b)$ and $x \in X$. Let $\tau \in(a, b), x \in X$, and $f=\chi_{[a, \tau]} x$. Given $\epsilon>0$ we define $\delta(t)=\epsilon$ if $t=\tau$ and $\delta(t)=(1 / 2)|\tau-t|$ if $t \neq \tau$; then for any $\delta$-fine tagged partition $\left\{\left(\left[t_{i-1}, t_{i}\right], \xi_{i}\right): i=1, \ldots, n\right\}$ of $[a, b], \tau$ is the tag of one subinterval, if $\xi_{i} \in(\tau, b), f\left(\xi_{i}\right)=0$, and $\int_{\tau}^{b} B(f, d g)=0$; otherwise

$$
\begin{aligned}
\sum_{i=1}^{n} & \| B\left(f\left(\xi_{i}\right), g\left(t_{i}\right)-g\left(t_{i-1}\right)\right) \\
- & {\left[B\left(x, g\left(t_{i}\right)\right)-B\left(x, g\left(t_{i-1}\right)\right)\right] \|_{Z} } \\
& =\sum_{i=1}^{n}\left\|B\left(f\left(\xi_{i}\right)-x, g\left(t_{i}\right)-g\left(t_{i-1}\right)\right)\right\|_{Z}=0<\epsilon .
\end{aligned}
$$

Hence $(H S) \int_{a}^{b} B(f, d g)=B(x, g(\tau))-B(x, g(a))$. The proofs of the cases $f=\chi_{[\tau, b]} x, f=\chi_{[a]} x$, and $f=\chi_{[b]} x$ are analogous.

Schwabik in [3] introduces the concept of vector-valued regulated functions; we shall only use the following characterization.

Theorem 23 (see [3, Prop. 2]). F : [a,b] $\rightarrow X$ is regulated if and only if it is the uniform limit of step functions.

Theorem 24. If $F:[a, b] \rightarrow X$ is regulated and $g:[a, b] \rightarrow$ $Y$ with $(\mathfrak{B}) \operatorname{var}_{a}^{b}(g)<\infty$, then the integral $(H S) \int_{a}^{b} B(F, d g)$ exists.

Proof. In as much as $F$ is regulated, there exists a sequence $F_{n}:[a, b] \rightarrow X, n=1,2, \ldots$, of step functions which converges uniformly to $F$, by Theorem 22; the integrals (HS) $\int_{a}^{b} B\left(F_{n}, d g\right)$ exist for each $n=1,2, \ldots$ The Uniform Convergence Theorem implies the existence of the integral (HS) $\int_{a}^{b} B(F, d g)$. 
Theorem 25 (integration by parts theorem). If $f:[a, b] \rightarrow X$ is Henstock integrable, $F$ its primitive, and $g:[a, b] \rightarrow Y$ with $(\mathfrak{B}) \operatorname{var}_{a}^{b}(g)<\infty$; then $(H) \int_{a}^{b} B(f, g)$ exists and

$$
\begin{aligned}
(H) \int_{a}^{b} B(f, g)= & B(F(b), g(b)) \\
& -(H S) \int_{a}^{b} B(F, d g) .
\end{aligned}
$$

Proof. Let $\epsilon>0$. Since $f$ is Henstock integrable, with $F$ its primitive, there exists a gauge $\delta_{1}$ such that if $P_{1}=\left\{\left(\left[t_{i-1}\right.\right.\right.$, $\left.\left.\left.t_{i}\right], \xi_{i}\right) ; i=1,2, \ldots, n\right\}$ is a $\delta_{1}$-fine tagged partition of $[a, b]$,

$$
\sum_{i=1}^{n}\left\|f\left(\xi_{i}\right)\left(t_{i}-t_{i-1}\right)-\left[F\left(t_{i}\right)-F\left(t_{i-1}\right)\right]\right\|_{X}<\epsilon .
$$

On the other hand, since $F$ is continuous, it is regulated, and by Theorem 24, (HS) $\int_{a}^{b} B(F, d g)$ exists; then there exists a gauge $\delta_{2}$ such that if $P_{2}=\left\{\left(\left[s_{j-1}, s_{j}\right], \eta_{j}\right) ; j=1,2, \ldots, m\right\}$ is a $\delta_{2}$-fine tagged partition of $[a, b]$,

$$
\begin{gathered}
\sum_{j=1}^{m} \| B\left(F\left(\eta_{j}\right), g\left(t_{j}\right)-g\left(t_{j-1}\right)\right) \\
-(H S) \int_{s_{j-1}}^{s_{j}} B(F, d g) \|_{Z}<\epsilon .
\end{gathered}
$$

We define a gauge $\delta^{*}$ by $\delta^{*}(t)=\min \left\{\delta_{1}(t), \delta_{2}(t)\right\}$. Let $P^{*}=$ $\left\{\left(\left[t_{k-1}, t_{k}\right], \xi_{k}\right), \quad k=i, \ldots, l\right\}$ be a $\delta^{*}$-fine tagged partition of $[a, b]$ and by the left-right process (see [13, Section 1, pp. 6]) we can assume that the tags are the left endpoint of each subinterval; then

$$
\begin{aligned}
& \sum_{k=1}^{l} \| B\left(f\left(t_{k}\right), g\left(t_{k}\right)\right)\left(t_{k}-t_{k-1}\right)-\left[B\left(F\left(t_{k}\right), g\left(t_{k}\right)\right)\right. \\
& \left.-B\left(F\left(t_{k-1}\right), g\left(t_{k-1}\right)\right)-(H S) \int_{t_{k-1}}^{k_{i}} B(F, d g)\right] \|_{Z} \\
& \quad \leq \sum_{k=1}^{l} \| B\left(f\left(t_{k}\right)\left(t_{k}-t_{k-1}\right)\right. \\
& \left.-\left[F\left(t_{k}\right)-F\left(t_{k-1}\right)\right], g\left(t_{k}\right)\right) \|_{Z} \\
& +\sum_{k=1}^{l} \| B\left(F\left(t_{k}\right), g\left(t_{k}\right)-g\left(t_{k-1}\right)\right)-(H S) \\
& \quad \cdot \int_{t_{k-1}}^{t_{k}} B(F, d g) \|_{Z} \leq \epsilon(\mathfrak{B}) \operatorname{var}_{a}^{b}(g)+\epsilon .
\end{aligned}
$$

As we can see, we have two types of integration by parts theorems, one is of the Stieltjes type and the other is nonStieltjes; it is possible to ask for the conditions so that the integral of the Stieltjes type becomes a non-Stieltjes; for that, we must do the following analysis:
(1) The essence in the proof of Theorem 19 is the derivative of the primitive of the function $t \rightarrow B(f(t)$, $G(t))$, that is, the Fundamental Theorem of Calculus.

(2) In Theorem 25 the Fundamental Theorem of Calculus does not apply because $g$ is not necessarily differentiable, and if it is, the primitive of $g^{\prime}$, in general, is not g.

(3) The condition of differentiability on a function $g$ : $[a, b] \rightarrow Y$ of strongly bounded variation is equivalent to $Y$ and has the Radon-Nikodým property (see Distel and Uhl [10, Chapter VII \$6]). Is it fulfilled with functions of strongly bounded $\mathfrak{B}$-variation?

Therefore, the condition of $g$ is one that ensures the Fundamental Theorem of Calculus, so we have the following theorems.

Theorem 26. Let $F:[a, b] \rightarrow X$ be continuous function and $g:[a, b] \rightarrow Y$ with $(\mathfrak{B}) \operatorname{var}_{a}^{b}(g)<\infty$ such that $g^{\prime}$ exists on $[a, b]$. Then

$$
(H) \int_{a}^{b} B\left(F, g^{\prime}\right)=(H S) \int_{a}^{b} B(F, d g) .
$$

Proof. (HS) $\int_{a}^{b} B(F, d g)$ exists by Theorem 24; then given $\epsilon>$ 0 , we have a gauge $\delta$ and $\left\{\left(\left[t_{i-1}, t_{i}\right], \xi_{i}\right): i=1, \ldots, n\right\}$ a $\delta$-fine tagged partition of $[a, b]$. Since $g^{\prime}$ exists, for every $t \in[a, b]$ there exists $\eta_{t}>0$ such that if $|t-s|<\eta_{t}$ then

$$
\left|\frac{g(t)-g(s)}{t-s}-g^{\prime}(t)\right|<\epsilon .
$$

We define a gauge $\delta_{1}:[a, b] \rightarrow(0, \infty)$ by $\delta_{1}(t)=\min \left\{\eta_{t}\right.$, $\delta(t)\}$. For all $\left\{\left(\left[t_{j-1}, t_{j}\right], \xi_{j}\right): j=1, \ldots, m\right\}$ which is $\delta_{1}$-fine tagged partition of $[a, b]$ and supposing that each tag is the left endpoint of its respective subinterval we have

$$
\begin{aligned}
& \sum_{j=1}^{m} \| B\left(F\left(t_{i}\right), g^{\prime}\left(t_{i}\right)\right)\left(t_{i}-t_{i-1}\right)-(H S) \\
& \cdot \int_{t_{i-1}}^{t_{i}} B(F, d g) \|_{Z} \\
& \leq \sum_{j=1}^{m} \| B\left(F\left(t_{i}\right), g^{\prime}\left(t_{i}\right)-\frac{g\left(t_{i}\right)-g\left(t_{i-1}\right)}{\left.t_{i}-t_{i-1}\right)}\right. \\
& \cdot\left(t_{i}-t_{i-1}\right)\left\|_{Z}+\sum_{j=1}^{m}\right\| B\left(F\left(t_{i}\right), g\left(t_{i}\right)-g\left(t_{i-1}\right)\right) \\
& -(H S) \int_{t_{i-1}}^{t_{i}} B(F, d g) \|_{Z}<\epsilon(M\|B\|(b-a)+1),
\end{aligned}
$$

where $M>0$ is the bound of $F$ due to it is continuous.

Obviously, we can change the condition over $g$ in Theorem 26 if we ask for strongly bounded variation and impose the Radon-Nikodým property on $Y$; either with these 
conditions or with those of Theorem 26 we can write equality (28) as

$$
(H) \int_{a}^{b} B(f, g)=B(F(b), g(b))-(H) \int_{a}^{b} B\left(F, g^{\prime}\right) \text {. }
$$

A function $G:[a, b] \rightarrow \mathbb{R}$ satisfies the Lipschitz condition if there exists $L>0$ such that $|G(t)-G(s)|<L|t-s|, s$, $t \in[a, b]$, and $G$ is of bounded slope variation (BSV) if

$$
\sum_{i=0}^{n-2}\left|\frac{G\left(t_{i+2}\right)-G\left(t_{i+1}\right)}{t_{i+2}-t_{i+1}}-\frac{G\left(t_{i+1}\right)-G\left(t_{i}\right)}{t_{i+1}-t_{i}}\right|
$$

is bounded for all divisions $a=t_{0}<t_{1}<\cdots<t_{n}=b$.

Lee in $[14$, p. 75$]$ proves that a function $G:[a, b] \rightarrow \mathbb{R}$ is the primitive (in the sense of Henstock) of a function of strongly bounded variation on $[a, b]$ if and only if $G$ satisfies the Lipschitz condition and is of bounded slope variation on $[a, b]$; this same characterization can be extended for our case (see the proof of [15, Thm. 10]). So we can see that the Fundamental Theorem of Calculus applies and we can restate the above integration by parts formula as follows.

Corollary 27. Let $f:[a, b] \rightarrow X$ be a function and $F$ its primitive. If $g:[a, b] \rightarrow Y$ satisfies the Lipschitz condition and is of bounded slope variation, then $(H) \int_{a}^{b} B(f, g)$ exists and

$$
(H) \int_{a}^{b} B(f, g)=\left.B(F, g)\right|_{a} ^{b}-(H) \int_{a}^{b} B\left(F, g^{\prime}\right) \text {. }
$$

\section{Representation Theorem}

Now, we will establish an important connection between the space of Henstock integrable functions and its dual space: a Riesz representation theorem.

Definition 28. Let $H([a, b], X)$ be the space of all Henstock integrable functions from $[a, b]$ to $X$. We define a norm on $H([a, b], X)$, called Alexiewicz norm (see e.g., [5] or [16]), by

$$
\|f\|_{A}=\sup \left\{\left\|(H) \int_{a}^{t} f\right\|_{X}: a \leq t \leq b\right\} .
$$

Theorem 29. Let $g:[a, b] \rightarrow Y$ be a function. If $(\mathfrak{B}) \operatorname{var}_{a}^{b}(g)$ $<\infty$, then

$$
T(f)=(H) \int_{a}^{b} B(f, g)
$$

defines a continuous linear operator on the space of Henstock integrable functions into $Z$ with

$$
\|T\| \leq\|B\|\|g(b)\|_{Y}+(\mathfrak{B}) \operatorname{var}_{a}^{b}(g) .
$$

Proof. Let $F:[a, b] \rightarrow X$ the primitive of $f$ and $\epsilon>0$; by Theorem 24 there exists a gauge $\delta$ such that if $\left\{\left(\left[t_{i-1}, t_{i}\right]\right.\right.$, $\left.\left.\xi_{i}\right) ; i=1, \ldots, n\right\}$ is $\delta$-fine,

$$
\begin{aligned}
& \sum_{i=1}^{n}\left\|B\left(F\left(\xi_{i}\right), g\left(t_{i}\right)-g\left(t_{i-1}\right)\right)-(H S) \int_{a}^{b} B(F, d g)\right\|_{Z} \\
& \quad<\epsilon .
\end{aligned}
$$

By the integration by parts theorem we have

$$
\begin{aligned}
& \|T(f)\|_{Z}=\left\|(H) \int_{a}^{b} B(f, g)\right\|_{Z}=\| B(F(b), g(b)) \\
& -(H S) \int_{a}^{b} B(F, d g)\left\|_{Z} \leq\right\| B(F(b), g(b)) \\
& -\sum_{i=1}^{n} B\left(F\left(\xi_{i}\right), g\left(t_{i}\right)-g\left(t_{i-1}\right)\right) \|_{Z} \\
& +\| \sum_{i=1}^{n} B\left(F\left(\xi_{i}\right), g\left(t_{i}\right)-g\left(t_{i-1}\right)\right) \\
& -(H S) \int_{a}^{b} B(F, d g)\left\|_{Z} \leq\right\| B(F(b), g(b)) \|_{Z} \\
& +\sum_{i=1}^{n}\left\|B\left(F\left(\xi_{i}\right), g\left(t_{i}\right)-g\left(t_{i-1}\right)\right)\right\|_{Z}+\epsilon \\
& \leq\|B(F(b), g(b))\|_{Z}+\|f\|_{A} \\
& \cdot \sum_{i=1,\left\|F\left(\xi_{i}\right)\right\|_{X} \neq 0}^{n}\left\|B\left(\frac{F\left(\xi_{i}\right)}{\left\|F\left(\xi_{i}\right)\right\|_{X}}, g\left(t_{i}\right)-g\left(t_{i-1}\right)\right)\right\|_{Z} \\
& +\epsilon \leq\|f\|_{A}\|B\|\|g(b)\|_{Y}+\|f\|_{A}(\mathfrak{B}) \operatorname{var}_{a}^{b}(g)+\epsilon .
\end{aligned}
$$

It follows that

$$
\frac{\|T(f)\|}{\|f\|_{A}} \leq\|B\|\|g(b)\|_{Y}+(\mathfrak{B}) \operatorname{var}_{a}^{b}(g) .
$$

So $T$ is bounded and continuous.

$L(X, Y)$ denotes the space of continuous linear operators from $X$ to $Y$ and $B: X \times L(X, Y) \rightarrow Y$ is the bilinear bounded operator given by $B(x, A)=A(x)$.

Theorem 30. Let $T: H([a, b], X) \rightarrow Y$ be a linear continuous operator defined on the space of the Henstock integrable functions. There exists a function $g:[a, b] \rightarrow L(X, Y)$ with $(\mathfrak{B}) \operatorname{var}_{a}^{b}(g)<\infty$ such that

$$
T(f)=(H) \int_{a}^{b} B(f, g)
$$

for every $f \in H([a, b], X)$.

Proof. For each $t \in[a, b]$ and $x \in X$ we have $\chi_{[a, t]} x \in$ $H([a, b], X)$. We define a function $\beta_{t}: X \rightarrow Y$ by

$$
\beta_{t}(x)=T\left(\chi_{[a, t]} x\right) .
$$

In this form, $\beta_{t}$ is linear and it is also continuous due to

$$
\left\|\beta_{t}(x)\right\|_{Y}=\left\|T\left(\chi_{[a, t]} x\right)\right\|_{Y} \leq M\|x\|_{X}
$$

where $M=\|T\|(b-a)$. 
We define a function $g:[a, b] \rightarrow L(X, Y)$ by

$$
g(t)=\beta_{t} \text {. }
$$

Now, $(\mathfrak{B}) \operatorname{var}_{a}^{b}(g)<\infty$, indeed, for every arbitrary partition $\left\{\left[t_{i-1}, t_{i}\right]: i=1, \ldots, n\right\}$ and for every $x_{i}, i=1, \ldots, n$, with $\left\|x_{i}\right\|_{X} \leq 1$,

$$
\begin{aligned}
\sum_{i=1}^{n} & \left\|B\left(x_{i}, g\left(t_{i}\right)-g\left(t_{i-1}\right)\right)\right\|_{Y} \\
& =\sum_{i=1}^{n}\left\|\beta_{t_{i}}\left(x_{i}\right)-\beta_{t_{i-1}}\left(x_{i}\right)\right\|_{Y} \\
& =\sum_{i=1}^{n}\left\|T\left(\chi_{\left[a, t_{i}\right]} x_{i}\right)-T\left(\chi_{\left[a, t_{i-1}\right]} x_{i}\right)\right\|_{Y} \\
& =\sum_{i=1}^{n}\left\|T\left(\chi_{\left[t_{i-1}, t_{i}\right]} x_{i}\right)\right\|_{Y} \leq\|T\| \sum_{i=1}^{n}\left\|x_{i}\right\|_{X}\left(t_{i}-t_{i-1}\right) \\
& \leq\|T\|(b-a) .
\end{aligned}
$$

Suppose that $s:[a, b] \rightarrow X$ is a step function; by Theorem 22 we have that the integral $(H S) \int_{a}^{b} B(s, d g)$ exists.

Given $f \in H([a, b], X)$, we take its primitive $F$ : $[a, b] \rightarrow X$. Since $F$ is continuous, there exists $\left(F_{n}\right)$ sequence of linear piecewise functions such that $F_{n} \rightarrow F$ uniformly on $[a, b]$. The integral $(H S) \int_{a}^{b} B\left(F_{n}, d g\right)$ exists for every $n$ by Theorem 24.

By the Uniform Convergence Theorem, the integral (HS) $\int_{a}^{b} B(F, d g)$ exists; hence

$$
\begin{aligned}
T(F) & =\lim _{n \rightarrow \infty} T\left(F_{n}\right)=\lim _{n \rightarrow \infty}(H S) \int_{a}^{b} B\left(F_{n}, d g\right) \\
& =(H S) \int_{a}^{b} B(F, d g) .
\end{aligned}
$$

Let $\left(f_{n}\right)$ be the sequence of derivatives of $F_{n} ; f_{n}$ is simple for each $n$, since $F_{n} \rightarrow F$ uniformly

$$
\left\|f_{n}-f\right\|_{A}=\left\|F_{n}-F\right\|_{\infty} \longrightarrow 0 ;
$$

by the continuity of $T$ and the Integration by parts Theorem 25 we have that

$$
\begin{aligned}
T(f) & =\lim _{n \rightarrow \infty} T\left(f_{n}\right)=\lim _{n \rightarrow \infty}(H) \int_{a}^{b} B\left(f_{n}, g\right) \\
& =(H) \int_{a}^{b} B(f, g) .
\end{aligned}
$$

Finally, we have the following representation theorem.

Corollary 31. $T: H([a, b], X) \rightarrow Y$ is a linear continuous operator if and only if there exists a function $g:[a, b] \rightarrow L(X$, $Y)$ with $(\mathfrak{B}) \operatorname{var}_{a}^{b}(g)<\infty$ such that

$$
T(f)=(H) \int_{a}^{b} B(f, g),
$$

for every $f \in H([a, b], X)$.
Consider $X$ a Banach space and $Y=X^{*}$ in the definition of strongly bounded $(\mathfrak{B})$-variation function; then this is equivalent to the definition of strongly bounded variation function, so we have the next result.

Corollary 32. $T$ is a linear continuous functional on $H([a, b]$, $X)$ if and only if there exists a function $g:[a, b] \rightarrow X^{*}$ and $V(g,[a, b])<\infty$ such that

$$
T(f)=(H) \int_{a}^{b} g(t) \circ f(t),
$$

for every $f \in H([a, b], X)$.

Hence, the dual space of $H([a, b], X)$ is isometrically isomorphic to the space of functions of strongly bounded variation.

Since the space of Henstock integrable real-valued functions coincides with the Kurzweil integrable functions we will name the integral as the integral of Henstock-Kurzweil.

As a particular case of Corollary 31 (with $X=L(X, Y)=$ $Y=\mathbb{R})$ we have obtained the following representation theorem for the space of Henstock-Kurzweil integrable functions.

Corollary 33. $T: H K([a, b], \mathbb{R}) \rightarrow \mathbb{R}$ is a linear continuous functional if and only if exists a function $g:[a, b] \rightarrow \mathbb{R}$, $V(g[a, b])<,\infty$, such that

$$
T(F)=(H K) \int_{a}^{b} f g,
$$

for every $f$ Henstock-Kurzweil integrable function.

Remarks 34. The result above was proved in [5] by Alexiewicz; later other proofs of the theorem arose, for example, those provided by Sargent and Lee (Theorem 4 in [17] and Theorem 12.7 in [14, pp. 76], resp.), who use different techniques from those used in this work; for example, HahnBanach Theorem is not necessary for the proof of Theorem 30.

The Integration by parts Corollary 27 yields a new representation theorem without using Stieltjes integral, which we shall establish next. The proof of the first result below is analogous to Theorem 29; using equality (35), we will only sketch the proof of the second result.

Theorem 35. If $g:[a, b] \rightarrow Y$ is of strongly bounded $\mathfrak{B}$ variation and differentiable and $g^{\prime}$ is bounded, then $T(f)=$ $(H) \int_{a}^{b} B(f, g)$ is a continuous linear operator on the space $H([a, b], X)$ into $Z$.

If $g:[a, b] \rightarrow Y$ is of bounded slope variation and satisfies the Lipschitz condition then the previous theorem is also true; we shall prove the second part in the new representation theorem.

Theorem 36. Let $T: H([a, b], X) \rightarrow Y$ be a linear continuous operator defined on the space of the Henstock integrable functions. There exists a function $g:[a, b] \rightarrow L(X, Y)$ of bounded slope variation and Lipschitz such that $T(f)=$ $(H) \int_{a}^{b} B(f, g)$, for every $f \in H([a, b], X)$. 
Proof. Let $x \in X$ and $F$ be the primitive of $f \in H([a, b], X)$. We define the function $\beta_{t}(x)=T\left(\chi_{[a, t]} x\right)$; this function is in $L(X, Y)$, and we define $g:[a, b] \rightarrow L(X, Y)$ by $g(t)=\beta_{t} ; g$ is of bounded slope variation and satisfies the Lipschitz condition (see the proof of [15, Thm. 10]); hence it is differentiable and $g^{\prime}$ is of strongly bounded variation. Following the proof of Theorem 30 using $g$ which is also of strongly bounded variation, by Theorem 26 the integral (HS) $\int_{a}^{b} B(F, d g)$ is equal to $(H) \int_{a}^{b} B\left(F, g^{\prime}\right)$ and by integration by parts Corollary 27, we have $T(f)=(H) \int_{a}^{b} B(f, g)$.

The last representation theorem identifies $H([a, b], X)$ with the space of primitives of the functions of strongly bounded variation, unlike the Corollary 31 which identifies it with the space of functions of strongly bounded $\mathfrak{B}$-variation.

\section{Conflicts of Interest}

The authors declare that they have no conflicts of interest.

\section{Acknowledgments}

This research has been able to see the light thanks to the help, comments, suggestions, and unconditional support of Professor Lee Peng Yee and the work team and the staff of MME/NIE of Nanyang Technological University in Singapore. The authors will be eternally grateful. This research has been supported by Conacyt, VIEP-BUAP, DGRIIA-BUAP, and the Academic Group of Mathematical Modeling and Differential Equations-FCFM-BUAP.

\section{References}

[1] R. Henstock, "A New Descriptive Definition of the Ward Integral," Journal of the London Mathematical Society, vol. s1-35, no. 1, pp. 43-48, 1960.

[2] S. S. Cao, "The Henstock integral for Banach-valued functions," Southeast Asian Bulletin of Mathematics, vol. 16, pp. 35-40, 1992.

[3] S. Schwabik, "Abstract Perron-Stieltjes integral," Mathematica Bohemica, vol. 121, no. 4, pp. 425-447, 1996.

[4] S. Schwabik, "A note on integration by parts for abstract PerronStieltjes integral," Math. Bohem, vol. 2001, no. 3, pp. 613-629, 2001.

[5] A. Alexiewicz, "Linear functionals on Denjoy-integrable functions," Colloquium Mathematicum, vol. 1, no. 4, pp. 289-293, 1947.

[6] B. Satco, "Solution tube method for impulsive periodic differential inclusions of first order," Nonlinear Analysis: Theory, Methods \& Applications, vol. 75, no. 1, pp. 260-269, 2012.

[7] V. Marraffa, "A descriptive characterization of the variational Henstock integral," in Proceedings of the International Mathematics Conference, vol. 22, pp. 73-84, Manila, Philippines, 1998.

[8] S. Schwabik and G. Ye, "Topics in Banach Space Integration, Real Analysis," in Real Analysis, vol. 10, World Scientific, Singapore, 2005.

[9] G. A. Monteiro, "On Functions of Bounded Semivariation," Real Analysis Exchange, vol. 40, no. 2, pp. 233-276, 2015.

[10] J. Diestel and J. J. Uhl, VectorMeasures, Mathematical surveys and monographs, American Mathematical Society, Mathematical surveys and monographs, 1977.
[11] Y. Guoju, W. Congxin, and L. P. Yee, "Integration by Parts for the Denjoy-Bochner Integral," Southeast Asian Bulletin of Mathematics, vol. 26, no. 4, pp. 693-700, 2003.

[12] G. A. Monteiro and M. Tvrdý, "On Kurzweil-Stieltjes integral in a Banach space," Mathematica Bohemica, vol. 137, no. 4, pp. 365-381, 2012.

[13] R. G. Bartle, A Modern Theory of Integration, vol. 32 of Graduate Studies in Mathematics, American Mathematical Society, 2001.

[14] P. Y. Lee, "Lanzhou Lectures on Henstock Integration," in Real Analysis, vol. 2, World Scientific, Singapore, 1989.

[15] R. M. Rey and P. Y. Lee, "A Representation Theorem for the Space of Henstock-Bochner integrable functions," Southeast Asian Bulletin of Mathematics, pp. 129-136, 1993.

[16] S. Saks, Theory of the Integral, PWN, Monografie Matematyczne, Warsaw, Poland, 2nd edition, 1937.

[17] W. L. C. Sargent, "On linear functionals in spaces of conditionally integrable functions," Quarterly Journal of Mathematics, vol. 1, no. 1, pp. 288-298, 1950. 


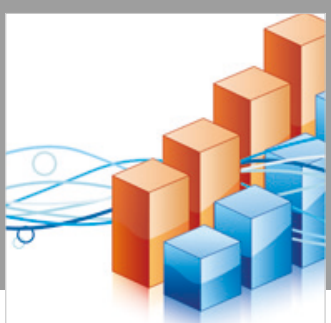

Advances in

Operations Research

\section{-n-m}
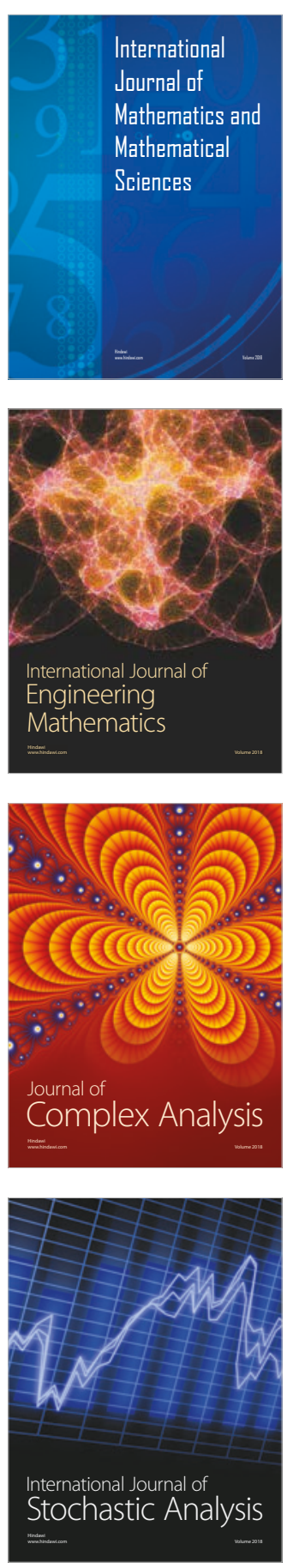
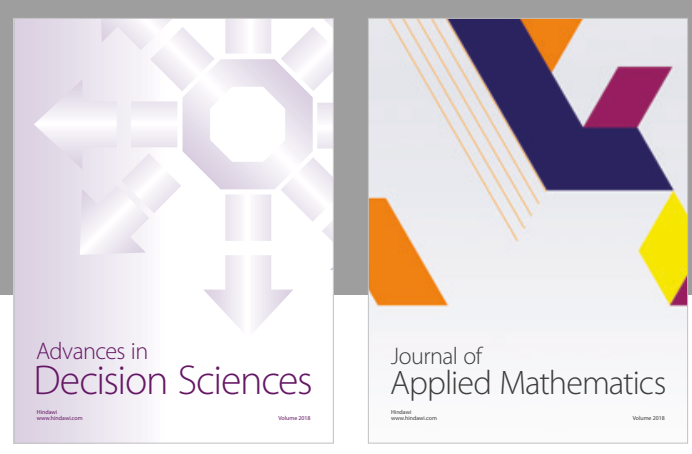

Journal of

Applied Mathematics
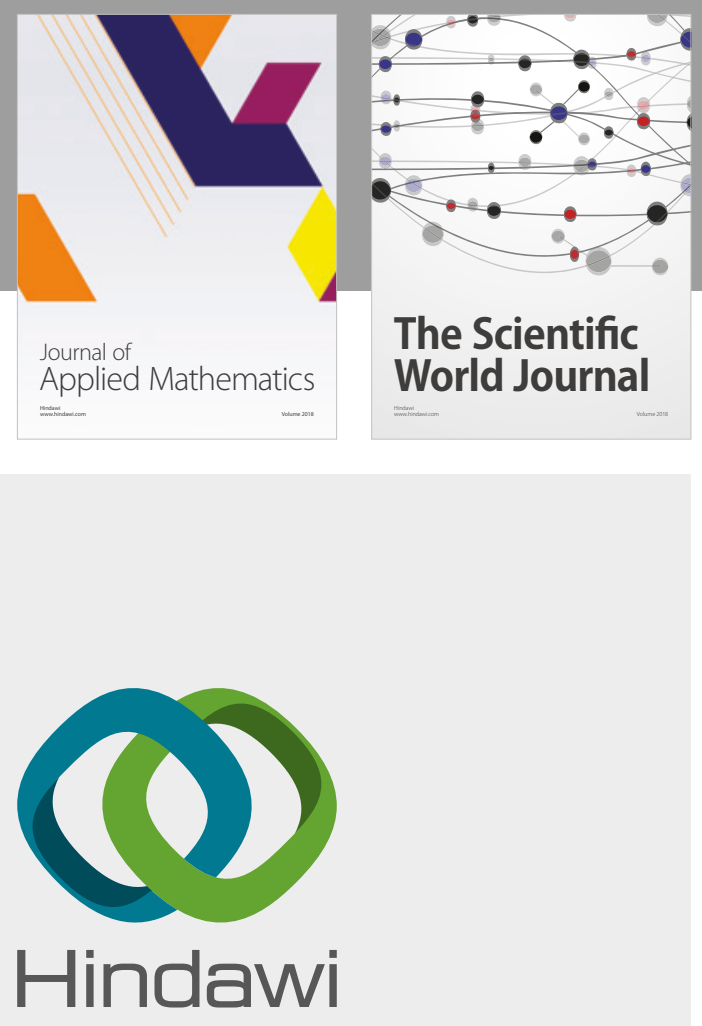

Submit your manuscripts at

www.hindawi.com

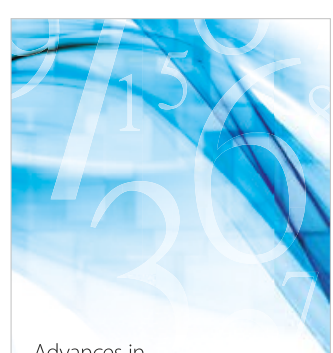

Advances in
Numerical Analysis
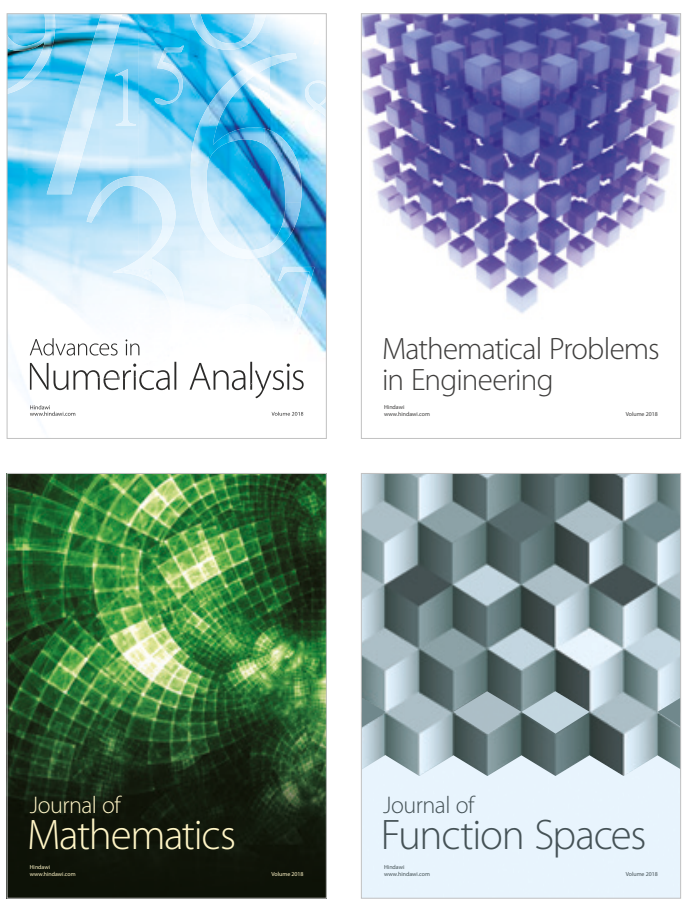

Mathematical Problems in Engineering

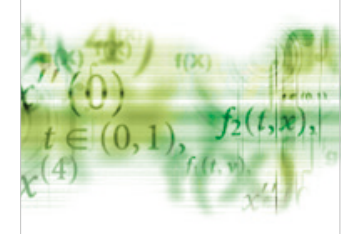

International Journal of

Differential Equations

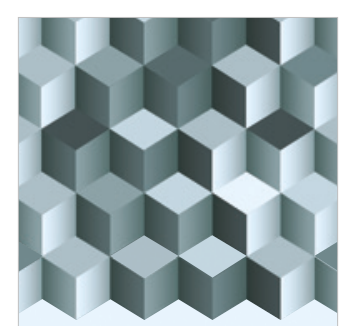

Journal of

Function Spaces

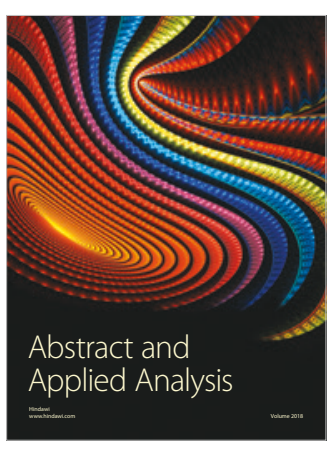

The Scientific

World Journal

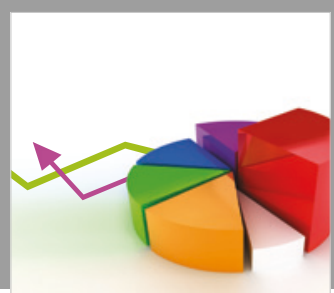

Journal of

Probability and Statistics
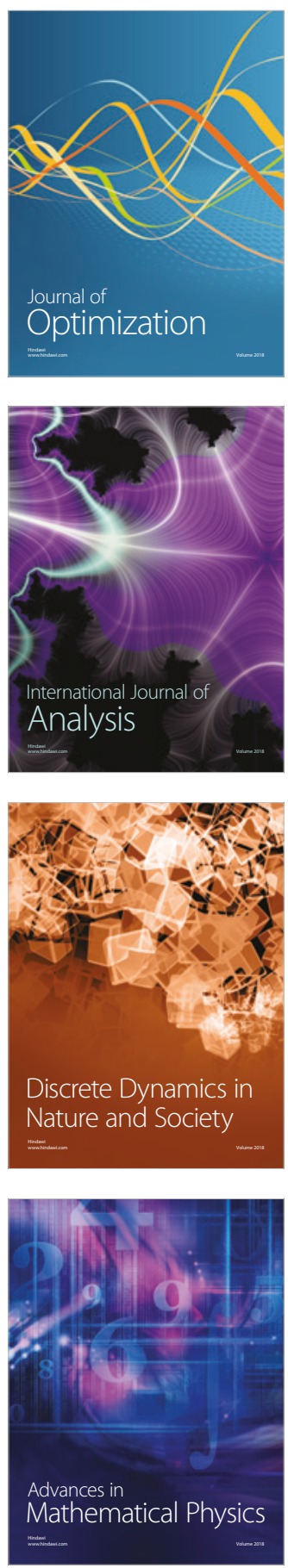